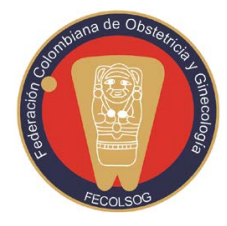

\title{
EL PARTO: EVENTO QUE EXIGE LA EXCELENCIA DE LA CALIDAD EN LOS SERVICIOS DE SALUD
}

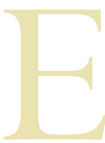

n este número de la revista se presenta un artículo de reflexión sobre las diferencias observadas en la calidad de la atención del parto que afectan de manera negativa a las gestantes con menos recursos económicos, las adolescentes y aquellas que presentan embarazos de alto riesgo.

Se parte del hecho de que idealmente el trabajo del parto y el parto deben ser "una vivencia satisfactoria" que incluye, además de brindar las mejores prácticas basadas en la evidencia científica, "respetar la fisiología del parto; intervenir solo lo necesario; identificar, comprender y respetar los aspectos socioculturales de la gestante; brindarle apoyo emocional; darle poder de decisión, y garantizar su autonomía y privacidad”. Estos deberían ser los criterios para evaluar la calidad en la atención del parto, en un concepto humanista amplio, que lleva a tener en cuenta, no solo los criterios clásicos de calidad, sino también dominios relacionados con los derechos humanos, la cultura de la gestante y principios éticos fundamentales.

Estos criterios han sido acuñados bajo el término de parto humanizado, expresión que fue acogida por la Guía de práctica clínica para la detección temprana de las anomalías durante el trabajo de parto, atención del parto normal y distócico del Ministerio de Salud y Protección Social de Colombia (1). Este incluye aspectos no solo relacionados con la atención médica y el cuidado de la gestante durante el proceso fisiológico de trabajo de parto y el parto, como por ejemplo: el control del dolor o el no uso de prácticas inadecuadas como el enema evacuador o el rasurado perineal, sino también el tomar en cuenta sus preferencias y temores, el respeto por su cultura y la garantía del derecho a la autonomía y privacidad en este momento especial de su vida (2). El no cumplimiento de estos criterios podría determinar un parto acompañado de una percepción de maltrato, lo que se ha consignado bajo la denominación de violencia obstétrica (3).

La mayoría de literatura disponible sobre parto humanizado proviene de Latinoamérica y del área de enfermería (4-8). El término humanización pretende, según la Real Academia Española (RAE), describir "el acto de hacer humano, familiar o afable algo" (9). Se intenta llamar la atención sobre las características que debe tener la atención del trabajo de parto y el parto, de captar emocionalmente el significado opuesto a atención deshumanizada o inhumana, lo que ha sido denominado por Hollander et al. como experiencias traumáticas durante el parto. Este autor menciona que aspectos tales como: la pérdida o ausencia de control, el sentir temor por la vida o la salud del bebé, el dolor o malestar físico intenso, la mala comunicación o no explicación al preguntar por lo que pasa con el resultado de las pruebas diagnósticas o el tratamiento, el sentir que no se es escuchado cuando se rechaza una intervención, o percibir que no se recibe ayuda de manera práctica y emocional, fueron los aspectos más frecuentemente mencionados como una experiencia traumática durante el parto, que se podría prevenir desde los cuidadores (10).

Desde nuestro punto de vista, sería más apropiado referirnos a una atención del parto con excelencia, en las acepciones que da la RAE, que incluyen: superior calidad o bondad que hace digno de singular aprecio y estimación algo, o tratamiento de respeto y cortesía que se da a las personas por su situación (9).

En el análisis de la calidad de la atención obstétrica el nivel mínimo aceptable se da por la no presencia 
de muertes maternas evitables, seguido por la menor morbilidad materna extrema prevenible, para llegar a un escalón más allá en el cual se aborda la atención segura, mediante la implementación de sistemas de análisis de incidentes clínicos y sistemas de gestión de riesgo destinados a reducir la presencia de los eventos adversos evitables. El escalón más alto en la evaluación de la calidad sería la atención del parto con excelencia, al cual se llega por la valoración de los criterios descritos, como la atención humanizada del parto.

La atención del parto con excelencia es un problema que interesa de manera individual a los obstetras, los médicos generales, las enfermeras del área de salud materna y perinatal, y a los anestesiólogos. También es de interés de las instituciones de servicios de salud, en donde se involucran desde los directores científicos y gerentes, hasta las operarias de servicios generales y personal que controla el ingreso a las instituciones de salud. Igualmente, atañe a las instituciones de educación superior en salud donde los profesores, estudiantes de pregrado y posgrado de las especialidades médicoquirúrgicas, maestrías y doctorados relacionados con la salud materna deben conocer sus principios y las teorías que los soportan.

El documento que induce a este editorial hace una aproximación general a este problema, y nos conduce a preguntarnos icuál es la situación de la excelencia en la atención del parto en Colombia? ¿Qué tanto se está cumpliendo con los principios de la excelencia en la atención del parto? ¿Es este cumplimiento igual para todas las gestantes?

En el año 2008, Conde Agudelo et al. (11) publicaron los resultados de una encuesta sobre el uso de intervenciones intraparto basadas en la evidencia realizada en ocho instituciones públicas y siete privadas en Cali, Colombia, en la que se revisaron las historias clínicas de gestantes de bajo riesgo y se realizaron entrevistas a profundidad a proveedores de servicios. Se encontró una frecuencia de uso de enema del 70\% y de rasurado del $75 \%$, y la presencia de un acompañante en el $14 \%$ de los partos. Los proveedores reconocían las ventajas de que la paciente recibiera apoyo durante el trabajo de parto, pero argüían múltiples razones para que este acompañamiento no se diera en la realidad en hospitales públicos, aunque sí en pacientes particulares en instituciones privadas.

Como se mencionó, en Colombia en el año 2013 fue publicada la Guía de atención del parto (1), en la que se encuentra como primera recomendación la adopción de los principios en los que se basa la atención humanizada del parto, y contiene otras recomendaciones orientadas a asegurar la atención bajo estos principios. Allí se recomienda: que la paciente esté acompañada por la persona que ella elija; la frecuencia sugerida de tactos vaginales y la garantía durante este examen de la privacidad y la dignidad; la buena comunicación cuando se requieran intervenciones o haya complicaciones; el derecho al control del dolor si la gestante lo solicita, con preferencia de las técnicas neuroaxiales; y se desaconseja el uso del enema y el rasurado perineal.

Es poca la información que se tiene sobre la implementación de estas recomendaciones en la atención del parto no complicado, y menos aún si estas son aplicadas de manera similar en las instituciones privadas y públicas, sin distinción del nivel socioeconómico, de la edad o condición clínica de las pacientes; sin embargo, sí hay información que nos orienta acerca de cómo podría ser la situación actual.

Por ejemplo, al revisar la Guía de manejo del trabajo de parto y parto publicada por la Secretaría de Salud de Bogotá en el 2013 (12), se menciona el control del dolor de la gestante durante el trabajo de parto mediante el uso de opiáceos y de "analgesia peridural en donde se encuentre disponible”. Esto hace suponer que en las instituciones públicas de Bogotá no se ofrece de manera habitual esta técnica, reconocida como la mejor para el control del dolor en el parto (13). Si esta situación se presenta en Bogotá, donde se tienen los mejores estándares de cuidado en atención materna en instituciones públicas a nivel nacional, surgen dudas sobre el control del dolor en hospitales de primer nivel ubicados en otras regiones del país, especialmente en las áreas rurales. Estas inequidades en la analgesia obstétrica en Colombia 
han sido planteadas previamente (14). Si la mayoría de partos en el país tienen atención institucional (98,6\%) (15), la analgesia obstétrica, incluyendo técnicas neuroaxiales, debería haber sido ofrecida y aplicada a esa misma proporción de gestantes. Las condiciones de infraestructura y oferta de servicios debería incluir la plena disponibilidad de la analgesia obstétrica a nivel nacional.

Otra barrera que se debe considerar para la implementación de la Guía de parto es la aproximación economicista que siguen las autoridades de salud nacionales y locales, así como los administradores de las instituciones, en la que se prioriza el punto de vista de la eficiencia sobre la efectividad, la seguridad, las preferencias y los derechos de los pacientes. Para el caso de Bogotá, la administración actual ha decidido centralizar la atención obstétrica en unos pocos hospitales (16), aumentando las barreras geográficas para la atención de la gestante, y desconociendo los principios de la atención del parto con excelencia respecto a las preferencias, la cultura y el derecho a la autonomía, medidas estas que de nuevo afectan a las gestantes de los estratos más pobres. Es otra muestra de la inequidad que afecta a la salud en Colombia y, en especial, a la salud materna (17).

\section{¿Cuál es el papel de las sociedades científicas y de la academia al respecto?}

Las sociedades científicas deberán jugar un papel muy importante a fin de lograr que se cumplan los principios de la atención del parto con excelencia y con equidad para todas las gestantes. En primer lugar, deberán sensibilizar a los especialistas en obstetricia, anestesia, pediatría, a los médicos generales y enfermeras, en la importancia de respetar los derechos de las gestantes en trabajo de parto, con base en un comportamiento ético, solidario y educado, más allá de las limitaciones institucionales, donde se ejerza el arte de la obstetricia basado en la mejor evidencia disponible. Por otra parte, deberán comprometerse a ejercer control social para que los entes gubernamentales emitan la reglamentación que permita aplicar lo contenido en las recomendaciones de la Guía, y para que los administradores y gerentes de las entidades promotoras de planes de beneficios y las instituciones (prestadores) que ofrecen servicios de atención materna efectúen los cambios en la estructura y en los procesos de atención inter e intrainstitucionales que promuevan los mejores estándares de calidad en la atención del trabajo de parto y del parto, en donde el maltrato obstétrico sea intolerable.

En cuanto a la academia, es muy importante que los estudiantes de pregrado y de posgrado relacionados con la atención del embarazo y el parto comprendan la importancia de respetar la autonomía de los pacientes; de reconocer, entender y respetar las diferencias culturales, y guardar la privacidad y la dignidad de la mujer durante el examen ginecológico, el cual solo se deberá hacer cuando sea estrictamente necesario, para vigilar la adecuada evolución del parto, y no por otras razones. Los estudiantes de pregrado deben conocer los derechos humanos, los derechos sexuales y reproductivos, los principios éticos que deben tener en cuenta para la atención médica con énfasis en la atención materna. Estos componentes deberán ser incluidos en los currículos respectivos, más allá de las consideraciones técnicas y científicas. La atención obstétrica resalta una multitud de dilemas éticos en cuanto a: el respeto por las personas, la garantía de la justicia y la equidad, el ejercicio de la autonomía, entre otros, que deben enfrentar todos los actores mencionados. Reconocer su presencia plantea retos en el quehacer de los profesionales, administradores, pacientes e instituciones, que podrían atenuar las inequidades existentes en la calidad de la atención obstétrica diferencial por estrato socioeconómico, régimen de atención en salud o ubicación geográfica, entre otras.

Por otra parte, se requieren estudios que evalúen la adherencia a las recomendaciones de la Guía que aseguran la atención del parto humanizado y las barreras en el acceso, en la estructura, en los procesos de atención que limitan su aplicación tanto en la atención privada como en los regímenes de aseguramiento contributivo y subsidiado, así como también 
la evaluación de indicadores que permitan verificar el cumplimiento de los estándares de la atención de trabajo de parto y parto con excelencia

La atención de la mujer gestante durante el control prenatal y el embarazo debe ser uno de los procesos de atención donde la evidencia derivada de la investigación clínica, la buena práctica, la experiencia, la solidaridad, el respeto del profesional y la ausencia de barreras deben juntarse en beneficio del fruto de la gestación y de la madre. Tolerancia CERO al maltrato obstétrico.

\section{Hernando Gaitán-Duarte, MD, MSc}

Editor

\section{Javier Eslava-Schmalbach, MD, MSc, PhD}

Editor invitado

Profesor Titular

Facultad de Medicina

Universidad Nacional de Colombia Sociedad

Colombiana de Anestesiología y Reanimación

(SCARE)

\section{REFERENCIAS}

1. Rubio-Romero JA, Ruiz-Parra AI, Martínez F, MuñozRestrepo J, Muñoz LA, Arévalo-Rodríguez I, et al. Guía de práctica clínica para la detección temprana de las anomalías durante el trabajo de parto, atención del parto normal y distócico. Rev Colomb Obstet Ginecol. 2013;64:379-424.

2. World Health Organization, Maternal and Newborn Health/Safe Motherhood Unit. Care in normal birth: a practical guide. Geneve: World Health Organization; 1996.

3. Diaz-Tello F. Invisible wounds: obstetric violence in the United States. Reprod Health Matters. 2016;24:56-64.

Beltran Salazar OA. The meaning of humanized nursing care for those participating in it: Importance of efforts of nurses and healthcare institutions. Investigación y Educación en Enfermería. 2016;34:18-28.

5. Binfa L, Pantoja L, Ortiz J, Gurovich M, Cavada G. Assessment of the implementation of the model of integrated and humanised midwifery health services in Santiago, Chile. Midwifery. 2013;29:1151-7.

6. Medeiros RM, Teixeira RC, Nicolini AB, Alvares AS, Correa AC, Martins DP. Humanized Care: insertion of obstetric nurses in a teaching hospital. Revista brasileira de enfermagem. 2016;69:1091-8.

7. Nora CR, Junges JR. [Humanization policy in primary health care: a systematic review]. Revista de saude publica. 2013;47:1186-200.

8. Serruya SJ, Cecatti JG, Lago T. [The Brazilian Ministry of Health's Program for Humanization of Prenatal and Childbirth Care: preliminary results]. Cadernos de saude publica. 2004;20:1281-9.

9. Real Academia Española. Diccionario de la Lengua Española. Madrid: Espasa; 2014 [visitado 2017 Jun 17]. Disponible en: http://dle.rae.es/.

10. Hollander MH, van Hastenberg E, van Dillen J, van Pampus MG, de Miranda E, Stramrood CAI. Preventing traumatic childbirth experiences: 2192 women's perceptions and views. Archives of women's mental health; 2017.

11. Conde-Agudelo A, Rosas-Bermudez A, Gulmezoglu AM. Evidence-based intrapartum care in Cali, Colombia: a quantitative and qualitative study. BJOG: An International Journal of Obstetrics and Gynaecology. 2008;115:1547-56.

12. Secretaría Distrital de Salud de Bogotá, Asociación Bogotana de Obstetricia y Ginecología (ASBOG). Guía de manejo de trabajo de parto, parto y sus complicaciones [visitado 2017 Jun 23]. Disponible en: http://www.saludcapital.gov.co/DDS/Paginas/ GuiasAtencion.aspx.

13. Anim-Somuah M, Smyth RM, Jones L. Epidural versus non-epidural or no analgesia in labour. The Cochrane database of systematic reviews. 2011(12):Cd000331.

14. Duarte Ortiz G, Navarro-Vargas JR, Eslava-Schmalbach J. Inequidad en el sistema de salud: el panorama de la analgesia obstétrica. Revista Colombiana de Anestesiología. 2013;41:215-7.

15. Ministerio de Salud y Protección Social. Informe Nacional de Calidad de la Atención en Salud. Bogotá: Ministerio de Salud y Protección Social; 2015.

16. Marín Correa A. Pediatras se quejan por traslado 
de unidad neonatal del Hospital Simón Bolívar. El Espectador; 2017.

17. Sandoval-Vargas YG, Eslava-Schmalbach JH. Inequality regarding maternal mortality in Colombian departments in 2000-2001, 2005-2006 and 2008-2009. Revista de Salud Pública. 2013;15:579-91. 